\title{
Two-Step Newton-Tikhonov Method for Hammerstein-Type Equations: Finite-Dimensional Realization
}

\author{
Santhosh George and Monnanda Erappa Shobha \\ Department of Mathematical and Computational Sciences, National Institute of Technology Karnataka, \\ Mangalore 575 025, India \\ Correspondence should be addressed to Santhosh George, sgeorge@nitk.ac.in
}

Received 26 December 2011; Accepted 26 January 2012

Academic Editors: A. Bellouquid, A. El-Sayed, A. J. Kearsley, J. Míguez, and J. Shen

Copyright (C) 2012 S. George and M. E. Shobha. This is an open access article distributed under the Creative Commons Attribution License, which permits unrestricted use, distribution, and reproduction in any medium, provided the original work is properly cited.

\begin{abstract}
Finite-dimensional realization of a Two-Step Newton-Tikhonov method is considered for obtaining a stable approximate solution to nonlinear ill-posed Hammerstein-type operator equations $K F(x)=f$. Here $F: D(F) \subseteq X \rightarrow X$ is nonlinear monotone operator, $K: X \rightarrow Y$ is a bounded linear operator, $X$ is a real Hilbert space, and $Y$ is a Hilbert space. The error analysis for this method is done under two general source conditions, the first one involves the operator $K$ and the second one involves the Fréchet derivative of $F$ at an initial approximation $x_{0}$ of the the solution $\hat{x}$ : balancing principle of Pereverzev and Schock (2005) is employed in choosing the regularization parameter and order optimal error bounds are established. Numerical illustration is given to confirm the reliability of our approach.
\end{abstract}

\section{Introduction}

Tikhonov's regularization (e.g., [1]) method has been used extensively to stabilize the approximate solution of nonlinear ill-posed problems. In recent years, increased emphasis has been placed on iterative regularization procedures $[2,3]$ for obtaining the approximate solution of such problems. In this paper, we examine the use of iterative regularization procedures for Hammerstein-type $[4,5]$ equations of the form

$$
K F(x)=f,
$$

where $F: D(F) \subseteq X \rightarrow X$, is nonlinear monotone operator, $F^{\prime}(\cdot)^{-1}$ does not exists, and $K: X \rightarrow Y$ is a bounded linear operator. Throughout this paper, $D(F)$ is the domain of $F, F^{\prime}(\cdot)$ is the Fréchet derivative of $F, X$ is a real Hilbert space, and $Y$ is a Hilbert space. The inner product and the corresponding norm are denoted by $\langle\cdot, \cdot\rangle$ and $\|\cdot\|$, respectively. 
Recall that [6] the operator $F$ is said to be a monotone operator if $\langle F(x)-F(y), x-y\rangle \geq$ 0 , for all $x, y \in D(F)$.

It is assumed throughout that $f^{\mathcal{\delta}} \in \mathcal{Y}$ are the available noisy data with

$$
\left\|f-f^{\delta}\right\| \leq \delta
$$

and $F$ possesses a uniformly bounded Fréchet derivative for each $x \in D(F)$ (cf. [7]), that is,

$$
\left\|F^{\prime}(x)\right\| \leq M, \quad x \in D(F)
$$

for some $M$.

Observe that the solution $x$ of (1.1) with $f^{\delta}$ in place of $f$ can be obtained by first solving

$$
K z=f^{\mathcal{E}}
$$

for $z$ and then solving the nonlinear problem

$$
F(x)=z
$$

In $[4,5,8]$ this was exploited. In $[4], z$ is approximated with $z_{\alpha}^{\delta}$ where

$$
z_{\alpha}^{\delta}=\left(K^{*} K+\alpha I\right)^{-1} K^{*} f^{\delta}, \quad \alpha>0, \delta>0,
$$

and then solve (1.5) iteratively using the following Newton-type procedure:

$$
x_{n+1, \alpha}^{\mathcal{S}}=x_{n, \alpha}^{\mathcal{\delta}}-F^{\prime}\left(x_{0}\right)^{-1}\left(F\left(x_{n, \alpha}^{\delta}\right)-z_{\alpha}^{\delta}\right)
$$

with $x_{0, \alpha}^{\delta}:=x_{0}$ and obtained local linear convergence. Here and below $x_{0} \in D(F)$ is a known initial approximation of the solution $\widehat{x}$ of (1.1) such that $\left\|x_{0}-\hat{x}\right\| \leq \rho$.

In [8], to solve (1.5), George and Kunhanandan used the iteration

$$
x_{n+1, \alpha}^{\delta}=x_{n, \alpha}^{\delta}-F^{\prime}\left(x_{n, \alpha}^{\delta}\right)^{-1}\left(F\left(x_{n, \alpha}^{\delta}\right)-z_{\alpha}^{\delta}\right), \quad x_{0, \alpha}^{\delta}:=x_{0}
$$

where

$$
z_{\alpha}^{\delta}=\left(K^{*} K+\alpha I\right)^{-1} K^{*}\left(f^{\mathcal{\delta}}-K F\left(x_{0}\right)\right)+F\left(x_{0}\right)
$$

and obtained local quadratic convergence.

A sequence $\left(x_{n}\right)$ in $X$ with $\lim x_{n}=x^{*}$ is said to be convergent of order $p>1$, if there exist positive reals $\beta$ and $\gamma$ such that, for all $n \in N$,

$$
\left\|x_{n}-x^{*}\right\| \leq \beta e^{-r p^{n}} .
$$


If a sequence $\left(x_{n}\right)$ satisfies $\left\|x_{n}-x^{*}\right\| \leq \beta q^{n}, 0<q<1$, then $\left(x_{n}\right)$ is said to be linearly convergent.

As in [8], it is assumed that the solution $\hat{x}$ of (1.1) satisfies

$$
\left\|F(\widehat{x})-F\left(x_{0}\right)\right\|=\min \left\{\left\|F(x)-F\left(x_{0}\right)\right\|: K F(x)=f, x \in D(F)\right\} .
$$

The regularization parameter $\alpha$ is chosen from a finite set

$$
D_{N}=\left\{\alpha_{i}: \alpha_{0}<\alpha_{1}<\alpha_{2}<\cdots<\alpha_{N}\right\}
$$

using the adaptive method considered by Pereverzev and Schock in [9].

In [10], Argyros and Hilout considered a method called Two-Step Directional Newton Method (TSDNM) for approximating a zero $x^{*}$ of a differentiable function $F$ defined on a convex subset $\Phi$ of a Hilbert space $H$ with values in $\mathbb{R}$. Motivated by TSDNM, in [11], we propose a Two-Step Newton-Tikhonov Methods (TSNTM) for solving (1.1).

In fact, in [11] we consider two cases of $F$, in the first case we assume that $F^{\prime}\left(x_{0}\right)^{-1}$ exist and in the second case we assume $F$ is monotone. In this paper we consider the finitedimensional realization of the second case, that is, $F$ is monotone. The finite-dimensional realization of the method and associated algorithm are proposed for which local-cubic convergence is established theoretically and validated numerically.

The organization of this paper is as follows. Section 2 deals with Discretized Tikhonov regularization and Section 3 investigates the convergence of the Discretized TSNTM. Section 4 discusses the algorithm and finally the paper ends with a numerical example in Section 5 .

\section{Discretized Tikhonov Regularization}

This section deals with discretized Tikhonov regularized solution $z_{\alpha}^{h, \delta}$ of (1.4) and (an a priori and an a posteriori) error estimate for $\left\|F(\widehat{x})-z_{\alpha}^{h, \delta}\right\|$ using an error estimate for $\left\|F(\widehat{x})-z_{\alpha}\right\|$ from [8].

The following assumption is used in [8] to obtain the error estimate.

Assumption 2.1. There exists a continuous, strictly monotonically increasing function $\varphi$ : $(0, a] \rightarrow(0, \infty)$ with $a \geq\|K\|^{2}$ satisfying

(i) $\lim _{\lambda \rightarrow 0} \varphi(\lambda)=0$,

(ii)

$$
\sup _{\lambda>0} \frac{\alpha \varphi(\lambda)}{\lambda+\alpha} \leq \varphi(\alpha) \quad \forall \lambda \in(0, a]
$$

(iii)

$$
F(\widehat{x})-F\left(x_{0}\right)=\varphi\left(K^{*} K\right) w
$$

for some $w \in X$ such that $\|w\| \leq 1$. 
Remark 2.2. The functions

$$
\varphi(\lambda):=\lambda^{\nu}, \quad \lambda>0
$$

for $0<v \leq 1$ and

$$
\varphi(\lambda)= \begin{cases}{\left[\ln \frac{1}{\lambda}\right]^{-p},} & 0<\lambda \leq e^{-(p+1)} \\ 0 & \text { otherwise }\end{cases}
$$

for $p \geq 0$ satisfy the above assumption (see [12]).

Theorem 2.3 (cf. [8], Theorem 4.3). Let $z_{\alpha}:=z_{\alpha}^{0}$ be as in (1.9) and Assumption 2.1 holds. Then

$$
\left\|F(\widehat{x})-z_{\alpha}\right\| \leq \varphi(\alpha) .
$$

Let $\left\{P_{h}\right\}_{h>0}$ be a family of orthogonal projections on $X$. Let

$$
\begin{gathered}
\varepsilon_{h}:=\left\|K\left(I-P_{h}\right)\right\|, \\
\tau_{h}:=\left\|F^{\prime}(x)\left(I-P_{h}\right)\right\|, \quad \forall x \in D(F)
\end{gathered}
$$

and $\left\{b_{h}: h>0\right\}$ is such that $\lim _{h \rightarrow 0}\left(\left\|\left(I-P_{h}\right) x_{0}\right\| / b_{h}\right)=0, \lim _{h \rightarrow 0}\left(\left\|\left(I-P_{h}\right) F\left(x_{0}\right)\right\| / b_{h}\right)=0$, and $\lim _{h \rightarrow 0} b_{h}=0$. We assume that $\varepsilon_{h} \rightarrow 0$ and $\tau_{h} \rightarrow 0$ as $h \rightarrow 0$. The above assumption is satisfied if, $P_{h} \rightarrow I$ pointwise and if $K$ and $F^{\prime}(x)$ are compact operators. Further we assume that $\varepsilon_{h}<\varepsilon_{0}, \tau_{h} \leq \tau_{0}, b_{h} \leq b_{0}$, and $\delta \in\left(0, \delta_{0}\right]$ where $\delta_{0}+\varepsilon_{0}<(2 /(2 M+3)) \sqrt{\alpha_{0}}$. The discretized Tikhonov regularization method for the regularized equation (1.4) consists of solving the equation

$$
\left(P_{h} K^{*} K P_{h}+\alpha P_{h}\right)\left(z_{\alpha}^{h, \delta}-P_{h} F\left(x_{0}\right)\right)=P_{h} K^{*}\left[f^{\delta}-K F\left(x_{0}\right)\right]
$$

Theorem 2.4. Suppose assumptions in Theorem 2.3 hold. Let $z_{\alpha}^{h, \delta}$ be as in (2.7) and $b_{h} \leq(\delta+$ $\left.\varepsilon_{h}\right) / \sqrt{\alpha}$. Then

$$
\left\|F(\widehat{x})-z_{\alpha}^{h, \delta}\right\| \leq C\left(\varphi(\alpha)+\left(\frac{\delta+\varepsilon_{h}}{\sqrt{\alpha}}\right)\right)
$$

where $C=(1 / 2) \max \{M \rho, 1\}+1$. 
Proof. Let $z_{\alpha}=\left(K^{*} K+\alpha I\right)^{-1} K^{*}\left(f-K F\left(x_{0}\right)\right)+F\left(x_{0}\right)$. Then

$$
\begin{aligned}
\left\|z_{\alpha}-z_{\alpha}^{h}\right\|= & \|\left(K^{*} K+\alpha I\right)^{-1} K^{*}\left(f-K F\left(x_{0}\right)\right) \\
& \quad-\left(P_{h} K^{*} K P_{h}+\alpha I\right)^{-1} P_{h} K^{*}\left(f-K F\left(x_{0}\right)\right)+F\left(x_{0}\right)-P_{h} F\left(x_{0}\right) \| \\
\leq & \left\|\left(P_{h} K^{*} K P_{h}+\alpha P_{h}\right)^{-1} P_{h} K^{*}\left(K P_{h}-K\right)\left(K^{*} K+\alpha I\right)^{-1} K^{*} K\left[F(\widehat{x})-F\left(x_{0}\right)\right]\right\| \\
& +\left\|\left(I-P_{h}\right) F\left(x_{0}\right)\right\| \\
\leq & \left\|F(\widehat{x})-F\left(x_{0}\right)\right\| \frac{\varepsilon_{h}}{2 \sqrt{\alpha}}+b_{h} \\
\leq & \left\|\int_{0}^{1} F^{\prime}\left(x_{0}+t\left(\widehat{x}-x_{0}\right)\right)\left(\widehat{x}-x_{0}\right) d t\right\| \frac{\varepsilon_{h}}{2 \sqrt{\alpha}}+b_{h} \\
\leq & M \rho \frac{\varepsilon_{h}}{2 \sqrt{\alpha}}+b_{h}, \\
\left\|z_{\alpha}^{h}-z_{\alpha}^{h, \delta}\right\|= & \left\|\left(P_{h} K^{*} K P_{h}+\alpha I\right)^{-1} P_{h} K^{*}\left(f-f^{\delta}\right)\right\| \\
\leq & \frac{\delta}{2 \sqrt{\alpha}} .
\end{aligned}
$$

Now the result follows from (2.9), Theorem 2.3 and the following triangle inequality:

$$
\left\|F(\widehat{x})-z_{\alpha, h}^{\delta}\right\| \leq\left\|F(\widehat{x})-z_{\alpha}\right\|+\left\|z_{\alpha}-z_{\alpha}^{h}\right\|+\left\|z_{\alpha}^{h}-z_{\alpha}^{h, \delta}\right\|
$$

\subsection{A Priori Choice of the Parameter}

Note that the estimate $\varphi(\alpha)+\left(\delta+\varepsilon_{h}\right) / \sqrt{ } \alpha$ in (2.8) is of optimal order for the choice $\alpha:=\alpha(\delta, h)$ which satisfies $\varphi(\alpha(\delta, h))=\left(\delta+\varepsilon_{h}\right) / \sqrt{\alpha(\delta, h)}$. Let $\psi(\lambda):=\lambda \sqrt{\varphi^{-1}(\lambda)}, 0<\lambda \leq a$. Then we have $\delta+\varepsilon_{h}=\sqrt{\alpha(\delta, h)} \varphi(\alpha(\delta, h))=\psi(\varphi(\alpha(\delta, h)))$ and

$$
\alpha(\delta, h)=\varphi^{-1}\left(\psi^{-1}\left(\delta+\varepsilon_{h}\right)\right)
$$

So the relation (2.8) leads to $\left\|F(\widehat{x})-z_{\alpha}^{h, \delta}\right\| \leq 2 C \psi^{-1}\left(\delta+\varepsilon_{h}\right)$.

\subsection{An Adaptive Choice of the Parameter}

In this subsection, we consider the balancing principle established by Pereverzev and Shock [9] for choosing the parameter $\alpha$. Let

$$
D_{N}=\left\{\alpha_{i}: 0<\alpha_{0}<\alpha_{1}<\alpha_{2}<\cdots<\alpha_{N}\right\}
$$

be the set of possible values of the parameter $\alpha$. 
Let

$$
\begin{gathered}
l:=\max \left\{i: \varphi\left(\alpha_{i}\right) \leq \frac{\delta+\varepsilon_{h}}{\sqrt{\alpha_{i}}}\right\}<N, \\
k=\max \left\{i: \alpha_{i} \in D_{N}^{+}\right\},
\end{gathered}
$$

where $D_{N}^{+}=\left\{\alpha_{i} \in D_{N}:\left\|z_{\alpha_{i}}^{\delta}-z_{\alpha_{j}}^{\delta}\right\| \leq 4 C\left(\delta+\varepsilon_{h}\right) / \sqrt{\alpha_{j}}, j=0,1,2, \ldots, i-1\right\}$.

We use the following theorem, proof of which is analogous to the proof of Theorem 4.3 in [8], for our error analysis.

Theorem 2.5 (cf. [8], Theorem 4.3). Let $l$ be as in (2.13), let $k$ be as in (2.14), and let $z_{\alpha_{k}}^{h, \delta}$ be as in (2.7) with $\alpha=\alpha_{k}:=\mu^{k} \alpha_{0}, \mu>1$. Then $l \leq k$ and

$$
\left\|F(\widehat{x})-z_{\alpha_{k}}^{h, \delta}\right\| \leq C\left(2+\frac{4 \mu}{\mu-1}\right) \mu \psi^{-1}\left(\delta+\varepsilon_{h}\right) .
$$

\section{Discretized Two-Step Newton Method (DTSNM)}

We need the following assumptions for the convergence of DTSNM and to obtain the error estimate.

Assumption 3.1 (cf. [7], Assumption 3 (A3)). There exists a constant $k_{0} \geq 0$ such that for every $x, u \in D(F)$ and $v \in X$ there exists an element $\Phi(x, u, v) \in X$ such that $\left[F^{\prime}(x)-F^{\prime}(u)\right] v=$ $F^{\prime}(u) \Phi(x, u, v),\|\Phi(x, u, v)\| \leq k_{0}\|v\|\|x-u\|$.

Assumption 3.2. There exists a continuous, strictly monotonically increasing function $\varphi_{1}$ : $(0, b] \rightarrow(0, \infty)$ with $b \geq\left\|F^{\prime}\left(x_{0}\right)\right\|$ satisfying

(i) $\lim _{\lambda \rightarrow 0} \varphi_{1}(\lambda)=0$,

(ii)

$$
\sup _{\lambda>0} \frac{\alpha \varphi_{1}(\lambda)}{\lambda+\alpha} \leq \varphi_{1}(\alpha) \quad \forall \lambda \in(0, b]
$$

(iii) there exists $v \in X$ with $\|v\| \leq 1$ (cf. [6]) such that

$$
x_{0}-\widehat{x}=\varphi_{1}\left(F^{\prime}\left(x_{0}\right)\right) v,
$$

(iv) for each $x \in B_{r}\left(x_{0}\right):=\left\{x:\left\|x-x_{0}\right\|<r\right\}$ there exists a bounded linear operator $G\left(x, x_{0}\right)$ (cf. [13]) such that

$$
F^{\prime}(x)=F^{\prime}\left(x_{0}\right) G\left(x, x_{0}\right)
$$

with $\left\|G\left(x, x_{0}\right)\right\| \leq K_{1}$. 
First we consider a DTSNM for approximating the zero $x_{c, \alpha_{k}}^{h, \delta}$ of

$$
P_{h}\left(F(x)+\frac{\alpha_{k}}{c}\left(x-x_{0}\right)\right)=P_{h} z_{\alpha_{k}}^{h, \delta}
$$

and then we show that $x_{c, \alpha_{k}}^{h, \delta}$ is an approximation to the solution $\widehat{x}$ of (1.1) where $c \leq \alpha_{k}$. For an initial guess $x_{0} \in X$ and for $R(x):=P_{h} F^{\prime}(x) P_{h}+\left(\alpha_{k} / c\right) P_{h}$, the DTSNM is defined as

$$
\begin{gathered}
y_{n, \alpha_{k}}^{h, \delta}=x_{n, \alpha_{k}}^{h, \delta}-R\left(x_{n, \alpha_{k}}^{h, \delta}\right)^{-1} P_{h}\left[F\left(x_{n, \alpha_{k}}^{h, \delta}\right)-z_{\alpha_{k}}^{h, \delta}+\frac{\alpha_{k}}{c}\left(x_{n, \alpha_{k}}^{h, \delta}-x_{0, \alpha_{k}}^{h, \delta}\right)\right], \\
x_{n+1, \alpha_{k}}^{h, \delta}=y_{n, \alpha_{k}}^{h, \delta}-R\left(x_{n, \alpha_{k}}^{h, \delta}\right)^{-1} P_{h}\left[F\left(y_{n, \alpha_{k}}^{h, \delta}\right)-z_{\alpha_{k}}^{h, \delta}+\frac{\alpha_{k}}{c}\left(y_{n, \alpha_{k}}^{h, \delta}-x_{0, \alpha_{k}}^{h, \delta}\right)\right],
\end{gathered}
$$

where $x_{0, \alpha_{k}}^{h, \delta}:=P_{h} x_{0}$. Note that with the above notation

$$
\begin{aligned}
\left\|R\left(x_{n, \alpha_{k}}^{h, \delta}\right)^{-1} P_{h} F^{\prime}\left(x_{n, \alpha_{k}}^{h, \delta}\right)\right\|= & \left\|\left(P_{h} F^{\prime}\left(x_{n, \alpha_{k}}^{h, \delta}\right) P_{h}+\frac{\alpha_{k}}{c} P_{h}\right)^{-1} P_{h} F^{\prime}\left(x_{n, \alpha_{k}}^{h, \delta}\right)\right\| \\
= & \left\|\left(P_{h} F^{\prime}\left(x_{n, \alpha_{k}}^{h, \delta}\right) P_{h}+\frac{\alpha_{k}}{c} P_{h}\right)^{-1} P_{h} F^{\prime}\left(x_{n, \alpha_{k}}^{h, \delta}\right)\left[P_{h}+I-P_{h}\right]\right\| \\
\leq & \left\|\left(P_{h} F^{\prime}\left(x_{n, \alpha_{k}}^{h, \delta}\right) P_{h}+\frac{\alpha_{k}}{c} P_{h}\right)^{-1} P_{h} F^{\prime}\left(x_{n, \alpha_{k}}^{h, \delta}\right) P_{h}\right\| \\
& +\left\|\left(P_{h} F^{\prime}\left(x_{n, \alpha_{k}}^{h, \delta}\right) P_{h}+\frac{\alpha_{k}}{c} P_{h}\right)^{-1} P_{h} F^{\prime}\left(x_{n, \alpha_{k}}^{h, \delta}\right)\left(I-P_{h}\right)\right\| \\
\leq & 1+\frac{\left\|P_{h} F^{\prime}\left(x_{n, \alpha_{k}}^{h, \delta}\right)\left(I-P_{h}\right)\right\|}{\alpha_{k} / c} \\
\leq & 1+\tau_{h} \leq 1+\tau_{0} .
\end{aligned}
$$

Let

$$
e_{n, \alpha_{k}}^{h, \delta}:=\left\|y_{n, \alpha_{k}}^{h, \delta}-x_{n, \alpha_{k}}^{h, \delta}\right\|, \quad \forall n \geq 0
$$

and let $k_{0}$ be such that

$$
\frac{k_{0}^{2}}{8}\left(4+3 k_{0}\left(1+\tau_{0}\right)\right)\left(1+\tau_{0}\right)^{2}<1
$$

Remark 3.3. Note that the above assumption is satisfied if we choose $k_{0}<\min \{1,(1 /(1+$ $\left.\left.\left.\tau_{0}\right)\right) \sqrt{8 / 4+3\left(1+\tau_{0}\right)}\right\}$.

Let $g:(0,1) \rightarrow(0,1)$ be the function defined by

$$
g(t)=\frac{k_{0}^{2}}{8}\left(4+3 k_{0}\left(1+\tau_{0}\right) t\right)\left(1+\tau_{0}\right)^{2} t^{2} \quad \forall t \in(0,1) .
$$


Let $\left\|\widehat{x}-x_{0}\right\| \leq \rho$, with

$$
\begin{aligned}
& \rho<\frac{1}{M}\left(1-\left(\frac{3}{2}+M\right) \frac{\delta_{0}+\varepsilon_{0}}{\sqrt{\alpha_{0}}}\right), \\
& \gamma_{\rho}:=M \rho+\left(\frac{3}{2}+M\right)\left(\frac{\varepsilon_{0}+\delta_{0}}{\sqrt{\alpha_{0}}}\right) .
\end{aligned}
$$

Theorem 3.4. Let $e_{n, \alpha_{k}}^{h, \delta}$ and $g$ be as in (3.8) and (3.10), respectively, and let $x_{n, \alpha_{k}}^{h, \delta}$ and $y_{n, \alpha_{k}}^{h, \delta}$ be as in (3.6) and (3.5), respectively, with $\delta \in\left(0, \delta_{0}\right], \alpha=\alpha_{k}$ and $\varepsilon_{h} \in\left(0, \varepsilon_{0}\right]$. Then the following holds:

(a)

$$
\left\|x_{n, \alpha_{k}}^{h, \delta}-y_{n-1, \alpha_{k}}^{h, \delta}\right\| \leq\left(1+\tau_{0}\right) \frac{k_{0} e_{n-1, \alpha_{k}}^{h, \delta}}{2}\left\|y_{n-1, \alpha_{k}}^{h, \delta}-x_{n-1, \alpha_{k}}^{h, \delta}\right\|
$$

(b)

$$
\left\|x_{n, \alpha_{k}}^{h, \delta}-x_{n-1, \alpha_{k}}^{h, \delta}\right\| \leq\left(1+\left(1+\tau_{0}\right) \frac{k_{0} e_{n-1, \alpha_{k}}^{h, \delta}}{2}\right)\left\|y_{n-1, \alpha_{k}}^{h, \delta}-x_{n-1, \alpha_{k}}^{h, \delta}\right\|
$$

(c)

$$
\left\|y_{n, \alpha_{k}}^{h, \delta}-x_{n, \alpha_{k}}^{h, \delta}\right\| \leq g\left(e_{n-1, \alpha_{k}}^{h, \delta}\right)\left\|y_{n-1, \alpha_{k}}^{h, \delta}-x_{n-1, \alpha_{k}}^{h, \delta}\right\|
$$

(d)

$$
g\left(e_{n, \alpha_{k}}^{h, \delta}\right) \leq g\left(\gamma_{\rho}\right)^{3^{n}}, \quad \forall n \geq 0
$$

(e)

$$
e_{n, \alpha_{k}}^{h, \delta} \leq g\left(\gamma_{\rho}\right)^{\left(3^{n}-1\right) / 2} \gamma_{\rho}, \quad \forall n \geq 0
$$

Proof. Observe that

$$
\begin{aligned}
x_{n, \alpha_{k}}^{h, \delta}-y_{n-1, \alpha_{k}}^{h, \delta}= & y_{n-1, \alpha_{k}}^{h, \delta}-x_{n-1, \alpha_{k}}^{h, \delta}-R\left(x_{n-1, \alpha_{k}}^{h, \delta}\right)^{-1} \\
& \times P_{h}\left(F\left(y_{n-1, \alpha_{k}}^{h, \delta}\right)-F\left(x_{n-1, \alpha_{k}}^{h, \delta}\right)+\frac{\alpha_{k}}{c}\left(y_{n-1, \alpha_{k}}^{h, \delta}-x_{n-1, \alpha_{k}}^{h, \delta}\right)\right) \\
= & R\left(x_{n-1, \alpha_{k}}^{h, \delta}\right)^{-1}\left[\begin{array}{l}
R\left(x_{n-1, \alpha_{k}}^{h, \delta}\right)\left(y_{n-1, \alpha_{k}}^{h, \delta}-x_{n-1, \alpha_{k}}^{h, \delta}\right) \\
\quad \\
\quad
\end{array} \quad P_{h}\left(F\left(y_{n-1, \alpha_{k}}^{h, \delta}\right)-F\left(x_{n-1, \alpha_{k}}^{h, \delta}\right)\right)-\frac{\alpha_{k}}{c}\left(y_{n-1, \alpha_{k}}^{h, \delta}-x_{n-1, \alpha_{k}}^{h, \delta}\right)\right]
\end{aligned}
$$


ISRN Applied Mathematics

$$
\begin{aligned}
= & R\left(x_{n-1, \alpha_{k}}^{h, \delta}\right)^{-1}\left[\left(P_{h} F^{\prime}\left(x_{n-1, \alpha_{k}}^{h, \delta}\right) P_{h}+\frac{\alpha_{k}}{c} P_{h}\right)\left(y_{n-1, \alpha_{k}}^{h, \delta}-x_{n-1, \alpha_{k}}^{h, \delta}\right)\right. \\
& \left.\quad-P_{h}\left(F\left(y_{n-1, \alpha_{k}}^{h, \delta}\right)-F\left(x_{n-1, \alpha_{k}}^{h, \delta}\right)\right)-\frac{\alpha_{k}}{c}\left(y_{n-1, \alpha_{k}}^{h, \delta}-x_{n-1, \alpha_{k}}^{h, \delta}\right)\right] \\
= & R\left(x_{n-1, \alpha_{k}}^{h, \delta}\right)^{-1} P_{h} \\
& \times \int_{0}^{1}\left[F^{\prime}\left(x_{n-1, \alpha_{k}}^{h, \delta}\right)-F^{\prime}\left(x_{n-1, \alpha_{k}}^{h, \delta}+t\left(y_{n-1, \alpha_{k}}^{h, \delta}-x_{n-1, \alpha_{k}}^{h, \delta}\right)\right)\right] P_{h}\left(y_{n-1, \alpha_{k}}^{h, \delta}-x_{n-1, \alpha_{k}}^{h, \delta}\right) d t .
\end{aligned}
$$

Now by Assumption 3.1 and (3.7) we have

$$
\begin{aligned}
\left\|x_{n, \alpha_{k}}^{h, \delta}-y_{n-1, \alpha_{k}}^{h, \delta}\right\| & \leq\left(1+\tau_{0}\right)\left\|\int_{0}^{1} \Phi\left(x_{n-1, \alpha_{k}}^{h, \delta}, x_{n-1, \alpha_{k}}^{h, \delta}+t\left(y_{n-1, \alpha_{k}}^{h, \delta}-x_{n-1, \alpha_{k}}^{h, \delta}\right), y_{n-1, \alpha_{k}}^{h, \delta}-x_{n-1, \alpha_{k}}^{h, \delta}\right) d t\right\| \\
& \leq\left(1+\tau_{0}\right) \frac{k_{0}}{2}\left\|y_{n-1, \alpha_{k}}^{h, \delta}-x_{n-1, \alpha_{k}}^{h, \delta}\right\|^{2} .
\end{aligned}
$$

This proves (a). Now (b) follows from (a) and the triangle inequality

$$
\left\|x_{n, \alpha_{k}}^{h, \delta}-x_{n-1, \alpha_{k}}^{h, \delta}\right\| \leq\left\|x_{n, \alpha_{k}}^{h, \delta}-y_{n-1, \alpha_{k}}^{h, \delta}\right\|+\left\|y_{n-1, \alpha_{k}}^{h, \delta}-x_{n-1, \alpha_{k}}^{h, \delta}\right\| .
$$

To prove (c) we observe that

$$
\begin{aligned}
& e_{n, \alpha_{k}}^{h, \delta}=\| x_{n, \alpha_{k}}^{h, \delta}-y_{n-1, \alpha_{k}}^{h, \delta}-R\left(x_{n, \alpha_{k}}^{h, \delta}\right)^{-1} P_{h}\left(F\left(x_{n, \alpha_{k}}^{h, \delta}\right)-z_{\alpha_{k}}^{h, \delta}+\frac{\alpha_{k}}{c}\left(x_{n, \alpha_{k}}^{h, \delta}-x_{0, \alpha_{k}}^{h, \delta}\right)\right) \\
&+R\left(x_{n-1, \alpha_{k}}^{h, \delta}\right)^{-1} P_{h}\left(F\left(y_{n-1, \alpha_{k}}^{h, \delta}\right)-z_{\alpha_{k}}^{h, \delta}+\frac{\alpha_{k}}{c}\left(y_{n-1, \alpha_{k}}^{h, \delta}-x_{0, \alpha_{k}}^{h, \delta}\right)\right) \| \\
&=\| x_{n, \alpha_{k}}^{h, \delta}-y_{n-1, \alpha_{k}}^{h, \delta}-R\left(x_{n, \alpha_{k}}^{h, \delta}\right)^{-1} P_{h}\left(F\left(x_{n, \alpha_{k}}^{h, \delta}\right)-F\left(y_{n-1, \alpha_{k}}^{h, \delta}\right)+\frac{\alpha_{k}}{c}\left(x_{n, \alpha}^{h, \delta}-y_{n-1, \alpha_{k}}^{h, \delta}\right)\right) \\
& \quad+\left[R\left(x_{n-1, \alpha_{k}}^{h, \delta}\right)^{-1}-R\left(x_{n, \alpha_{k}}^{h, \delta}\right)^{-1}\right] \times P_{h}\left(F\left(y_{n-1, \alpha_{k}}^{h, \delta}\right)-z_{\alpha_{k}}^{h, \delta}+\frac{\alpha_{k}}{c}\left(y_{n-1, \alpha_{k}}^{h, \delta}-x_{0, \alpha_{k}}^{h, \delta}\right)\right) \| \\
&:=\Gamma_{1}+\Gamma_{2},
\end{aligned}
$$

where

$$
\begin{gathered}
\Gamma_{1}:=\left\|x_{n, \alpha_{k}}^{h, \delta}-y_{n-1, \alpha_{k}}^{h, \delta}-R\left(x_{n, \alpha_{k}}^{h, \delta}\right)^{-1} P_{h}\left(F\left(x_{n, \alpha_{k}}^{h, \delta}\right)-F\left(y_{n-1, \alpha_{k}}^{h, \delta}\right)+\frac{\alpha_{k}}{c}\left(x_{n, \alpha}^{h, \delta}-y_{n-1, \alpha_{k}}^{h, \delta}\right)\right)\right\|, \\
\Gamma_{2}:=\left\|\left[R\left(x_{n-1, \alpha_{k}}^{h, \delta}\right)^{-1}-R\left(x_{n, \alpha_{k}}^{h, \delta}\right)^{-1}\right] P_{h}\left(F\left(y_{n-1, \alpha_{k}}^{h, \delta}\right)-z_{\alpha_{k}}^{h, \delta}+\frac{\alpha_{k}}{c}\left(y_{n-1, \alpha_{k}}^{h, \delta}-x_{0, \alpha_{k}}^{h, \delta}\right)\right)\right\| .
\end{gathered}
$$


Note that

$$
\begin{aligned}
\Gamma_{1} & =\left\|R\left(x_{n, \alpha_{k}}^{h, \delta}\right)^{-1}\left[P_{h} F^{\prime}\left(x_{n, \alpha_{k}}^{h, \delta}\right) P_{h}\left(x_{n, \alpha_{k}}^{h, \delta}-y_{n-1, \alpha_{k}}^{h, \delta}\right)-P_{h}\left(F\left(x_{n, \alpha_{k}}^{h, \delta}\right)-F\left(y_{n-1, \alpha_{k}}^{h, \delta}\right)\right)\right]\right\| \\
& =\left\|R\left(x_{n, \alpha_{k}}^{h, \delta}\right)^{-1} P_{h} \int_{0}^{1}\left[F^{\prime}\left(x_{n, \alpha_{k}}^{h, \delta}\right)-F^{\prime}\left(y_{n-1, \alpha_{k}}^{h, \delta}+t\left(x_{n, \alpha_{k}}^{h, \delta}-y_{n-1, \alpha_{k}}^{h, \delta}\right)\right)\right] \times P_{h}\left(x_{n, \alpha_{k}}^{h, \delta}-y_{n-1, \alpha_{k}}^{h, \delta}\right) d t\right\| \\
& \leq\left(1+\tau_{0}\right)\left\|\int_{0}^{1} \Phi\left(x_{n, \alpha_{k}}^{h, \delta}, y_{n-1, \alpha_{k}}^{h, \delta}+t\left(x_{n, \alpha_{k}}^{h, \delta}-y_{n-1, \alpha_{k}}^{h, \delta}\right), x_{n, \alpha_{k}}^{h, \delta}-y_{n-1, \alpha_{k}}^{h, \delta}\right) d t\right\| \\
& \leq\left(1+\tau_{0}\right) \frac{k_{0}}{2}\left\|x_{n, \alpha_{k}}^{h, \delta}-y_{n-1, \alpha_{k}}^{h, \delta}\right\|^{2} .
\end{aligned}
$$

The last but one step follows from Assumption 3.1 and (3.7). Similarly one can prove that

$$
\Gamma_{2} \leq\left(1+\tau_{0}\right) k_{0}\left\|x_{n, \alpha_{k}}^{h, \delta}-x_{n-1, \alpha_{k}}^{h, \delta}\right\|\left\|x_{n, \alpha_{k}}^{h, \delta}-y_{n-1, \alpha_{k}}^{h, \delta}\right\| .
$$

Thus from (3.20), (3.22), (3.23), (a) and (b) we have

$$
\begin{aligned}
e_{n, \alpha_{k}}^{h, \delta} \leq & \left(1+\tau_{0}\right)^{2}\left(\frac{k_{0}^{2}}{2}+\frac{3 k_{0}^{3}\left(1+\tau_{0}\right)}{8}\left\|y_{n-1, \alpha_{k}}^{h, \delta}-x_{n-1, \alpha_{k}}^{h, \delta}\right\|\right) \\
& \times\left\|y_{n-1, \alpha_{k}}^{h, \delta}-x_{n-1, \alpha_{k}}^{h, \delta}\right\|^{3} \\
\leq & g\left(e_{n-1, \alpha_{k}}^{h, \delta}\right) e_{n-1, \alpha_{k}}^{h, \delta} .
\end{aligned}
$$

Again since for $\mu \in(0,1), g(\mu t) \leq \mu^{2} g(t)$, for all $t \in(0,1)$, by (3.24) we have

$$
\begin{gathered}
g\left(e_{n, \alpha_{k}}^{h, \delta}\right) \leq g\left(e_{0, \alpha_{k}}^{h, \delta}\right)^{3^{n}} \\
e_{n, \alpha_{k}}^{h, \delta} \leq g^{3}\left(e_{n-2, \alpha_{k}}^{h, \delta}\right) e_{n-1, \alpha_{k}}^{h, \delta} \leq g^{3}\left(e_{n-2, \alpha_{k}}^{h, \delta}\right) g^{3}\left(e_{n-3, \alpha_{k}}^{h, \delta}\right) e_{n-2, \alpha_{k}}^{h, \delta} \cdots g\left(e_{0, \alpha_{k}}^{h, \delta}\right) e_{0, \alpha_{k}}^{h, \delta} \\
\leq \\
g\left(e_{0, \alpha_{k}}^{h, \delta}\right)^{3^{n-1}+3^{n-2}+\cdots+1} e_{0, \alpha_{k}}^{h, \delta} \\
\leq \\
g\left(e_{0, \alpha_{k}}^{h, \delta}\right)^{\left(3^{n}-1\right) / 2} e_{0, \alpha_{k}}^{h, \delta}
\end{gathered}
$$

provided $e_{n, \alpha_{k}}^{h, \delta}<1$, for all $n \geq 0$. From (3.26) it is clear that, $e_{n, \alpha_{k}}^{h, \delta} \leq 1$ if $e_{0, \alpha_{k}}^{h, \delta} \leq 1$. This can be seen as follows:

$$
\begin{aligned}
e_{0, \alpha_{k}}^{h, \delta} & =\left\|y_{0, \alpha_{k}}^{h, \delta}-x_{0, \alpha_{k}}^{h, \delta}\right\|=\left\|y_{0, \alpha_{k}}^{h, \delta}-P_{h} x_{0}\right\| \\
& =\left\|\left(P_{h} F^{\prime}\left(P_{h} x_{0}\right) P_{h}+\frac{\alpha_{k}}{c}\right)^{-1} P_{h}\left(F\left(P_{h} x_{0}\right)-z_{\alpha_{k}}^{h, \delta}\right)\right\| \\
& \leq\left\|F\left(P_{h} x_{0}\right)-z_{\alpha_{k}}^{h}\right\|+\left\|z_{\alpha_{k}}^{h}-z_{\alpha_{k}}^{h, \delta}\right\|,
\end{aligned}
$$


ISRN Applied Mathematics

$$
\begin{aligned}
\left\|F\left(P_{h} x_{0}\right)-z_{\alpha_{k}}^{h}\right\| \leq & \left\|F\left(P_{h} x_{0}\right)-F\left(x_{0}\right)\right\|+\left\|F\left(x_{0}\right)-z_{\alpha_{k}}\right\|+\left\|z_{\alpha_{k}}-z_{\alpha_{k}}^{h}\right\| \\
\leq & \left\|\int_{0}^{1} F^{\prime}\left(x_{0}+t\left(P_{h} x_{0}-x_{0}\right)\right)\left(P_{h} x_{0}-x_{0}\right) d t\right\| \\
& \quad+\left\|\left(K^{*} K+\alpha_{k} I\right)^{-1} K^{*} K\left(F(\widehat{x})-F\left(x_{0}\right)\right)\right\|+\left\|z_{\alpha_{k}}-z_{\alpha_{k}}^{h}\right\| \\
\leq & M b_{h}+\left\|F(\widehat{x})-F\left(x_{0}\right)\right\|+\left\|z_{\alpha_{k}}-z_{\alpha_{k}}^{h}\right\| \\
\leq & M b_{h}+M \rho+\left\|z_{\alpha_{k}}-z_{\alpha_{k}}^{h}\right\| .
\end{aligned}
$$

Therefore by (3.27) and (2.9) we have

$$
\begin{aligned}
e_{0, \alpha_{k}}^{h, \delta} & \leq(M+1) b_{h}+\left(1+\frac{\varepsilon_{h}}{2 \sqrt{\alpha_{k}}}\right) M \rho+\frac{\delta}{2 \sqrt{\alpha_{k}}} \\
& \leq(M+1) \frac{\varepsilon_{h}+\delta}{\sqrt{\alpha_{k}}}+M \rho+\frac{1}{2} \max \{M \rho, 1\} \frac{\varepsilon_{0}+\delta_{0}}{\sqrt{\alpha_{0}}} \\
& \leq(M+1) \frac{\varepsilon_{0}+\delta_{0}}{\sqrt{\alpha_{0}}}+M \rho+\frac{\varepsilon_{0}+\delta_{0}}{2 \sqrt{\alpha_{0}}} \\
& \leq \gamma_{\rho}<1 .
\end{aligned}
$$

Now since $g$ is monotonic increasing and $e_{0, \alpha_{k}}^{h, \delta} \leq \gamma_{\rho}$, we have $g\left(e_{0, \alpha_{k}}^{h, \delta}\right) \leq g\left(\gamma_{\rho}\right)$. This completes the proof of the theorem.

Theorem 3.5. Let $r=\left(1 /\left(1-g\left(\gamma_{\rho}\right)\right)+\left(1+\tau_{0}\right)\left(k_{0} / 2\right)\left(\gamma_{\rho} /\left(1-g\left(\gamma_{\rho}\right)^{2}\right)\right)\right) \gamma_{\rho}$ and the assumptions of Theorem 3.4 hold. Then $x_{n, \alpha_{k}}^{h, \delta}, y_{n, \alpha_{k}}^{h, \delta} \in B_{r}\left(P_{h} x_{0}\right)$, for all $n \geq 0$.

Proof. Note that by (b) of Theorem 3.4 we have

$$
\begin{aligned}
\left\|x_{1, \alpha_{k}}^{h, \delta}-P_{h} x_{0}\right\| & \leq\left[1+\left(1+\tau_{0}\right) \frac{k_{0}}{2} e_{0, \alpha_{k}}^{h, \delta}\right] e_{0, \alpha_{k}}^{h, \delta} \\
& \leq\left[1+\left(1+\tau_{0}\right) \frac{k_{0}}{2} \gamma_{\rho}\right] \gamma_{\rho} \\
& \leq r,
\end{aligned}
$$

that is, $x_{1} \in B_{r}\left(P_{h} x_{0}\right)$. Again note that by (3.29) and (c) of Theorem 3.4 we have

$$
\begin{aligned}
\left\|y_{1, \alpha_{k}}^{h, \delta}-P_{h} x_{0}\right\| & \leq\left\|y_{1, \alpha_{k}}^{h, \delta}-x_{1, \alpha_{k}}^{h, \delta}\right\|+\left\|x_{1, \alpha_{k}}^{h, \delta}-P_{h} x_{0}\right\| \\
& \leq\left(1+g\left(e_{0, \alpha_{k}}^{h, \delta}\right)+\left(1+\tau_{0}\right) \frac{k_{0}}{2} e_{0, \alpha_{k}}^{h, \delta}\right) e_{0, \alpha_{k}}^{h, \delta} \\
& \leq\left(1+g\left(\gamma_{\rho}\right)+\left(1+\tau_{0}\right) \frac{k_{0}}{2} \gamma_{\rho}\right) r_{\rho} \\
& \leq r
\end{aligned}
$$


that is, $y_{1, \alpha_{k}}^{h, \delta} \in B_{r}\left(P_{h} x_{0}\right)$. Further by (3.29) and (b) of Theorem 3.4 we have

$$
\begin{aligned}
\left\|x_{2, \alpha_{k}}^{h, \delta}-P_{h} x_{0}\right\| \leq & \left\|x_{2, \alpha_{k}}^{h, \delta}-x_{1, \alpha_{k}}^{h, \delta}\right\|+\left\|x_{1, \alpha_{k}}^{h, \delta}-P_{h} x_{0}\right\| \\
\leq & \left(1+\left(1+\tau_{0}\right) \frac{k_{0}}{2} e_{1, \alpha_{k}}^{h, \delta}\right) e_{1, \alpha_{k}}^{h, \delta} \\
& +\left(1+\left(1+\tau_{0}\right) \frac{k_{0}}{2} e_{0, \alpha_{k}}^{h, \delta}\right) e_{0, \alpha_{k}}^{h, \delta} \\
\leq & \left(1+g\left(e_{0, \alpha_{k}}^{h, \delta}\right)+\left(1+\tau_{0}\right) \frac{k_{0}}{2} e_{0, \alpha_{k}}^{h, \delta}\left(1+g\left(e_{0, \alpha_{k}}^{h, \delta}\right)^{2}\right)\right) e_{0, \alpha_{k}}^{h, \delta} \\
\leq & r .
\end{aligned}
$$

The last but one step follows from the monotonicity of $g$ and (3.28).

And by (3.31) and (c) of Theorem 3.4 we have

$$
\begin{aligned}
\left\|y_{2, \alpha_{k}}^{h, \delta}-P_{h} x_{0}\right\| & \leq\left\|y_{2, \alpha_{k}}^{h, \delta}-x_{2, \alpha_{k}}^{h, \delta}\right\|+\left\|x_{2, \alpha_{k}}^{h, \delta}-P_{h} x_{0}\right\| \\
& \leq g\left(e_{1, \alpha_{k}}^{h, \delta}\right) e_{1, \alpha_{k}}^{h, \delta}+\left(1+g\left(e_{0, \alpha_{k}}^{h, \delta}\right)+\left(1+\tau_{0}\right) \frac{k_{0}}{2} e_{0, \alpha_{k}}^{h, \delta}\left(1+g\left(e_{0, \alpha_{k}}^{h, \delta}\right)^{2}\right)\right) e_{0, \alpha_{k}}^{h, \delta} \\
& \leq g\left(e_{0, \alpha_{k}}^{h, \delta}\right)^{4} e_{0, \alpha_{k}}^{h, \delta}+\left(1+g\left(e_{0, \alpha_{k}}^{h, \delta}\right)+\left(1+\tau_{0}\right) \frac{k_{0}}{2} e_{0, \alpha_{k}}^{h, \delta}\left(1+g\left(e_{0, \alpha_{k}}^{h, \delta}\right)^{2}\right)\right) e_{0, \alpha_{k}}^{h, \delta} \\
& \leq\left(1+g\left(\gamma_{\rho}\right)+g\left(\gamma_{\rho}\right)^{2}+\left(1+\tau_{0}\right) \frac{k_{0}}{2} \gamma_{\rho}\left(1+g\left(\gamma_{\rho}\right)^{2}\right)\right) r_{\rho} \\
& \leq r,
\end{aligned}
$$

that is, $x_{2, \alpha^{\prime}}^{h, \delta}, y_{2, \alpha_{k}}^{h, \delta} \in B_{r}\left(P_{h} x_{0}\right)$. Continuing this way one can prove that $x_{n, \alpha_{k}}^{h, \delta} y_{n, \alpha_{k}}^{h, \delta} \in B_{r}\left(P_{h} x_{0}\right)$, for all $n \geq 0$. This completes the proof.

The main result of this section is the following theorem.

Theorem 3.6. Let $y_{n, \alpha_{k}}^{h, \delta}$ and $x_{n, \alpha_{k}}^{h, \delta}$ be as in (3.5) and (3.6), respectively, and let assumptions of Theorem 3.5 hold. Then $\left(x_{n, \alpha_{k}}^{h, \delta}\right)$ is a Cauchy sequence in $B_{r}\left(P_{h} x_{0}\right)$ and converges to $x_{c, \alpha_{k}}^{h, \delta} \in \overline{B_{r}\left(P_{h} x_{0}\right)}$. Further $P_{h}\left[F\left(x_{c, \alpha_{k}}^{h, \delta}\right)+\left(\alpha_{k} / c\right)\left(x_{c, \alpha_{k}}^{h, \delta}-x_{0}\right)\right]=P_{h} z_{\alpha_{k}}^{h, \delta}$ and

$$
\left\|x_{n, \alpha_{k}}^{h, \delta}-x_{c, \alpha_{k}}^{h, \delta}\right\| \leq \overline{C_{0}} e^{-\gamma_{1} 3^{n}}
$$

where $\overline{C_{0}}=\left(1 /\left(1-g\left(\gamma_{\rho}\right)^{3}\right)+\left(1+\tau_{0}\right)\left(k_{0} \gamma_{\rho} / 2\right)\left(1 /\left(1-\left(g\left(\gamma_{\rho}\right)^{2}\right)^{3}\right)\right) g\left(\gamma_{\rho}\right)^{3^{n}}\right) \gamma_{\rho}$ and $\gamma_{1}=-\log g\left(\gamma_{\rho}\right)$. 
Proof. Using the relation (b) and (e) of Theorem 3.4 and (3.28), we obtain

$$
\begin{aligned}
\left\|x_{n+m, \alpha_{k}}^{h, \delta}-x_{n, \alpha_{k}}^{h, \delta}\right\| & \leq \sum_{i=0}^{i=m-1}\left\|x_{n+i+1, \alpha_{k}}^{h, \delta}-x_{n+i, \alpha_{k}}^{h, \delta}\right\| \\
& \leq \sum_{i=0}^{i=m-1}\left(1+\left(1+\tau_{0}\right) \frac{k_{0} e_{n+i, \alpha_{k}}^{h, \delta}}{2}\right) e_{n+i, \alpha_{k}}^{h, \delta} \\
& \leq \sum_{i=0}^{i=m-1}\left(1+\left(1+\tau_{0}\right) \frac{k_{0} e_{0, \alpha_{k}}^{h, \delta}}{2} g\left(e_{0, \alpha_{k}}^{h, \delta}\right)^{3^{n+i}}\right) g\left(e_{0, \alpha_{k}}^{h, \delta}\right)^{3^{n+i}} e_{0, \alpha_{k}}^{h, \delta} \\
& \leq\left[\left(1+g\left(\gamma_{\rho}\right)^{3}+g\left(\gamma_{\rho}\right)^{3^{2}}+\cdots+g\left(\gamma_{\rho}\right)^{3^{m}}\right)+\left(1+\tau_{0}\right) \frac{k_{0} \gamma_{\rho}}{2}\right. \\
& \leq \overline{C_{0}} g\left(\gamma_{\rho}\right)^{3^{n}} \\
& \leq \overline{C_{0}} e^{-\gamma_{1} 3^{n}} .
\end{aligned}
$$

Thus $\left(x_{n, \alpha_{k}}^{h, \delta}\right)$ is a Cauchy sequence in $B_{r}\left(P_{h} x_{0}\right)$ and hence it converges, say, to $x_{c, \alpha_{k}}^{h, \delta} \in \overline{B_{r}\left(P_{h} x_{0}\right)}$. Observe that from (3.5)

$$
\begin{aligned}
\left\|P_{h}\left(F\left(x_{n, \alpha_{k}}^{h, \delta}\right)-z_{\alpha_{k}}^{h, \delta}\right)+\frac{\alpha_{k}}{c}\left(x_{n, \alpha_{k}}^{h, \delta}-P_{h} x_{0}\right)\right\|= & \left\|R\left(x_{n, \alpha_{k}}^{h, \delta}\right)\left(x_{n, \alpha_{k}}^{h, \delta}-y_{n, \alpha_{k}}^{h, \delta}\right)\right\| \\
\leq & \left\|R\left(x_{n, \alpha_{k}}^{h, \delta}\right)\right\|\left\|y_{n, \alpha_{k}}^{h, \delta}-x_{n, \alpha_{k}}^{h, \delta}\right\| \\
\leq & \left(\left\|P_{h} F^{\prime}\left(x_{n, \alpha_{k}}^{h, \delta}\right) P_{h}\right\|+\frac{\alpha_{k}}{c}\right) e_{n, \alpha_{k}}^{h, \delta} \\
\leq & \left(\left\|P_{h} F^{\prime}\left(x_{n, \alpha_{k}}^{h, \delta}\right) P_{h}\right\|+\frac{\alpha_{k}}{c}\right) \\
& \times g\left(e_{0, \alpha_{k}}^{h, \delta}\right)^{3^{n}} e_{0, \alpha_{k}}^{h, \delta} \\
\leq & \left(M+\frac{\alpha_{k}}{c}\right) g\left(\gamma_{\rho}\right)^{3^{n}} \gamma_{\rho} .
\end{aligned}
$$

Now by letting $n \rightarrow \infty$ in (3.35) we obtain $P_{h} F\left(x_{c, \alpha_{k}}^{h, \delta}\right)+\left(\alpha_{k} / c\right)\left(x_{c, \alpha_{k}}^{h, \delta}-P_{h} x_{0}\right)=P_{h} z_{\alpha_{k}}^{h, \delta}$. This completes the proof.

Hereafter we assume that $r<1 / k_{0}$ and $K_{1}<\left(1-k_{0} r\right) /(1-c)$. The proof of the following theorem is analogous to the proof of Theorem 3.14 in [11] but for the sake of completeness we give the proof.

Theorem 3.7 (cf. [11], Theorem 3.14). Suppose $x_{c, \alpha_{k}}^{\delta}$ is the solution of

$$
F(x)+\frac{\alpha_{k}}{c}\left(x-x_{0}\right)=z_{\alpha_{k}}^{\delta}
$$


and Assumptions 3.1 and 3.2 holds, then

$$
\left\|\widehat{x}-x_{c, \alpha_{k}}^{\delta}\right\| \leq \frac{\varphi_{1}\left(\alpha_{k}\right)+(2+4 \mu /(\mu-1)) \mu \psi^{-1}\left(\delta+\varepsilon_{h}\right)}{1-(1-c) K_{1}-k_{0} r} .
$$

Proof. Note that $c\left(F\left(x_{c, \alpha_{k}}^{\delta}\right)-z_{\alpha_{k}}^{\delta}\right)+\alpha_{k}\left(x_{c, \alpha_{k}}^{\delta}-x_{0}\right)=0$, so

$$
\begin{aligned}
\left(F^{\prime}\left(x_{0}\right)+\alpha_{k} I\right)\left(x_{c, \alpha_{k}}^{\delta}-\widehat{x}\right)= & \left(F^{\prime}\left(x_{0}\right)+\alpha_{k} I\right)\left(x_{c, \alpha_{k}}^{\delta}-\widehat{x}\right) \\
& -c\left(F\left(x_{c, \alpha_{k}}^{\delta}\right)-z_{\alpha_{k}}^{\delta}\right)-\alpha_{k}\left(x_{c, \alpha}^{\delta}-x_{0}\right) \\
= & \alpha_{k}\left(x_{0}-\widehat{x}\right)-c\left(F(\widehat{x})-z_{\alpha_{k}}^{\delta}\right)+F^{\prime}\left(x_{0}\right)\left(x_{c, \alpha_{k}}^{\delta}-\widehat{x}\right) \\
& -c\left[F\left(x_{c, \alpha_{k}}^{\delta}\right)-F(\widehat{x})\right] .
\end{aligned}
$$

Thus

$$
\begin{aligned}
\left\|x_{c, \alpha_{k}}^{\delta}-\widehat{x}\right\| \leq & \left\|\alpha_{k}\left(F^{\prime}\left(x_{0}\right)+\alpha_{k} I\right)^{-1}\left(x_{0}-\widehat{x}\right)\right\|+\left\|\left(F^{\prime}\left(x_{0}\right)+\alpha_{k} I\right)^{-1} c\left(F(\widehat{x})-z_{\alpha_{k}}^{\delta}\right)\right\| \\
& +\left\|\left(F^{\prime}\left(x_{0}\right)+\alpha_{k} I\right)^{-1}\left[F^{\prime}\left(x_{0}\right)\left(x_{c, \alpha_{k}}^{\delta}-\widehat{x}\right)-c\left(F\left(x_{c, \alpha_{k}}^{\delta}\right)-F(\widehat{x})\right)\right]\right\| \\
\leq & \left\|\alpha_{k}\left(F^{\prime}\left(x_{0}\right)+\alpha_{k} I\right)^{-1}\left(x_{0}-\widehat{x}\right)\right\|+\left\|F(\widehat{x})-z_{\alpha_{k}}^{\delta}\right\|+\Gamma,
\end{aligned}
$$

where $\Gamma:=\|\left(F^{\prime}\left(x_{0}\right)+\alpha_{k} I\right)^{-1} \int_{0}^{1}\left[F^{\prime}\left(x_{0}\right)-c F^{\prime}\left(\widehat{x}+t\left(x_{c, \alpha_{k}}^{\delta}-\widehat{x}\right)\right]\left(x_{c, \alpha_{k}}^{\delta}-\widehat{x}\right) d t \|\right.$. So by Assumption 3.2, we obtain

$$
\begin{aligned}
\Gamma \leq & \left\|\left(F^{\prime}\left(x_{0}\right)+\alpha_{k} I\right)^{-1} \int_{0}^{1}\left[F^{\prime}\left(x_{0}\right)-F^{\prime}\left(\widehat{x}+t\left(x_{c, \alpha_{k}}^{\delta}-\widehat{x}\right)\right)\right] \times\left(x_{c, \alpha_{k}}^{\delta}-\widehat{x}\right) d t\right\| \\
& +(1-c)\left\|\left(F^{\prime}\left(x_{0}\right)+\alpha_{k} I\right)^{-1} F^{\prime}\left(x_{0}\right) \times \int_{0}^{1} G\left(\widehat{x}+t\left(x_{c, \alpha_{k}}^{\delta}-\widehat{x}\right), x_{0}\right)\left(x_{c, \alpha_{k}}^{\delta}-\hat{x}\right) d t\right\| \\
& \leq k_{0} r\left\|x_{c, \alpha_{k}}^{\delta}-\hat{x}\right\|+(1-c) K_{1}\left\|x_{c, \alpha_{k}}^{\delta}-\hat{x}\right\|
\end{aligned}
$$

and hence by (3.39) and (3.40) we have

$$
\begin{aligned}
\left\|x_{c, \alpha_{k}}^{\delta}-\widehat{x}\right\| & \leq \frac{\left\|\alpha_{k}\left(F^{\prime}\left(x_{0}\right)+\alpha_{k} I\right)^{-1}\left(x_{0}-\widehat{x}\right)\right\|+\left\|F(\widehat{x})-z_{\alpha_{k}}^{\delta}\right\|}{1-(1-c) K_{1}-k_{0} r} \\
& \leq \frac{\varphi_{1}\left(\alpha_{k}\right)+(2+4 \mu /(\mu-1)) \mu \psi^{-1}\left(\delta+\varepsilon_{h}\right)}{1-(1-c) K_{1}-k_{0} r} .
\end{aligned}
$$

This completes the proof of the theorem. 
Theorem 3.8. Suppose $x_{c, \alpha_{k}}^{h, \delta}$ is the solution of (3.4) and Assumption 2.1 and Theorem 3.7 hold. In addition if $\tau_{0}<1$, then

$$
\left\|x_{c, \alpha_{k}}^{h, \delta}-x_{c, \alpha_{k}}^{\delta}\right\| \leq \frac{2}{1-\tau_{0}}\left(\frac{\delta+\varepsilon_{h}}{\sqrt{\alpha_{k}}}\right) .
$$

Proof. Suppose $x_{c, \alpha_{k}}^{\mathcal{\delta}}$ and $x_{c, \alpha_{k}}^{h, \delta}$ are the solutions of (3.36) and (3.4), respectively, then by (3.36) we have,

$$
P_{h} F\left(x_{c, \alpha_{k}}^{\delta}\right)+\frac{\alpha_{k}}{c}\left(P_{h} x_{c, \alpha_{k}}^{h, \delta}-P_{h} x_{0}\right)=P_{h} z_{\alpha_{k}}^{\delta}
$$

So from (3.4) and (3.43),

$$
P_{h}\left[F\left(x_{c, \alpha_{k}}^{h, \delta}\right)-F\left(x_{c, \alpha_{k}}^{\delta}\right)\right]+\frac{\alpha_{k}}{c} P_{h}\left(x_{c, \alpha_{k}}^{h, \delta}-x_{c, \alpha_{k}}^{\delta}\right)=P_{h}\left(z_{\alpha_{k}}^{h, \delta}-z_{\alpha_{k}}^{\delta}\right) .
$$

Let $M_{f}=\int_{0}^{1} F^{\prime}\left(x_{c, \alpha_{k}}^{\delta}+t\left(x_{c, \alpha_{k}}^{h, \delta}-x_{c, \alpha_{k}}^{\delta}\right)\right) d t$. Then by (3.44) we have

$$
P_{h}\left[M_{f}\left(x_{c, \alpha_{k}}^{h, \delta}-x_{c, \alpha_{k}}^{\delta}\right)\right]+\frac{\alpha_{k}}{c} P_{h}\left(x_{c, \alpha_{k}}^{h, \delta}-x_{c, \alpha_{k}}^{\delta}\right)=P_{h}\left(z_{\alpha_{k}}^{h, \delta}-z_{\alpha_{k}}^{\delta}\right)
$$

and hence

$$
\begin{aligned}
\left\|x_{c, \alpha_{k}}^{h, \delta}-x_{c, \alpha_{k}}^{\delta}\right\| & \leq\left\|z_{\alpha_{k}}^{h, \delta}-z_{\alpha_{k}}^{\delta}\right\|+\left\|M_{f}\left(P_{h}-I\right)\right\|\left\|x_{c, \alpha_{k}}^{h, \delta}-x_{c, \alpha_{k}}^{\delta}\right\| \\
& \leq\left\|z_{\alpha_{k}}^{h, \delta}-z_{\alpha_{k}}^{\delta}\right\|+\tau_{0}\left\|x_{c, \alpha_{k}}^{h, \delta}-x_{c, \alpha_{k}}^{\delta}\right\| .
\end{aligned}
$$

Thus

$$
\begin{aligned}
\left\|x_{c, \alpha_{k}}^{h, \delta}-x_{c, \alpha_{k}}^{\delta}\right\| & \leq \frac{1}{1-\tau_{0}}\left\|z_{\alpha_{k}}^{h, \delta}-z_{\alpha_{k}}^{\delta}\right\| \\
& \leq \frac{1}{1-\tau_{0}}\left[\left\|z_{\alpha_{k}}^{h, \delta}-z_{\alpha_{k}}^{h}\right\|+\left\|z_{\alpha_{k}}^{h}-z_{\alpha_{k}}\right\|+\left\|z_{\alpha_{k}}-z_{\alpha_{k}}^{\delta}\right\|\right] .
\end{aligned}
$$

Now the result follows from (2.9), (3.47) and the relation

$$
\left\|z_{\alpha_{k}}-z_{\alpha_{k}}^{\delta}\right\| \leq \frac{\delta}{2 \sqrt{\alpha_{k}}}
$$

The following theorem is a consequence of Theorems 3.6, 3.7, and 3.8.

Theorem 3.9. Let $x_{n, \alpha_{k}}^{h, \delta}$ be as in (3.6) and let assumptions in Theorems 3.6, 3.7 and 3.8 hold. Then

$$
\left\|\widehat{x}-x_{n_{k}, \alpha_{k}}^{h, \delta}\right\| \leq \overline{C_{0}} e^{-\gamma_{1} 3^{n}}+\frac{\varphi_{1}\left(\alpha_{k}\right)+(2+4 \mu /(\mu-1)) \mu \psi^{-1}\left(\delta+\varepsilon_{h}\right)}{1-(1-c) K_{1}-k_{0} r}+\frac{2}{1-\tau_{0}}\left(\frac{\delta+\varepsilon_{h}}{\sqrt{\alpha_{k}}}\right),
$$

where $\overline{C_{0}}$ and $\gamma_{1}$ are as in Theorem 3.6. 
Theorem 3.10. Let $x_{n, \alpha_{k}}^{h, \delta}$ be as in (3.6) and let assumptions in Theorem 3.9 hold. Further let $\varphi_{1}\left(\alpha_{k}\right) \leq$ $\varphi\left(\alpha_{k}\right)$ and

$$
n_{k}:=\min \left\{n: e^{-\gamma_{1} 3^{n}} \leq \frac{\delta+\varepsilon_{h}}{\sqrt{\alpha_{k}}}\right\}
$$

Then

$$
\left\|\widehat{x}-x_{n, \alpha_{k}}^{h, \delta}\right\|=O\left(\psi^{-1}\left(\delta+\varepsilon_{h}\right)\right) .
$$

\section{Algorithm}

Note that for $i, j \in\{0,1,2, \ldots, N\}$,

$$
z_{\alpha_{i}}^{h, \delta}-z_{\alpha_{j}}^{h, \delta}=\left(\alpha_{j}-\alpha_{i}\right)\left(P_{h} K^{*} K P_{h}+\alpha_{j} I\right)^{-1}\left(P_{h} K^{*} K P_{h}+\alpha_{i} I\right)^{-1} P_{h} K^{*}\left(f^{\delta}-K F\left(x_{0}\right)\right) .
$$

Therefore the balancing principle algorithm associated with the choice of the parameter specified in Section 2 involves the following steps:

Step 1. Choose $\alpha_{0}$ such that $\delta_{0}+\varepsilon_{0}<2 \sqrt{\alpha_{0}} /(2 M+3)$ and $\mu>1$.

Step 2. $\alpha_{i}=\mu^{2 i} \alpha_{0}$.

Step 3. Solve for $w_{i}$ :

$$
\left(P_{h} K^{*} K P_{h}+\alpha_{i} I\right) w_{i}=P_{h} K^{*}\left(f^{\delta}-K F\left(x_{0}\right)\right)
$$

Step 4. Solve for $j<i, z_{i j}^{h, \mathcal{S}}:\left(P_{h} K^{*} K P_{h}+\alpha_{j} I\right) z_{i j}^{h, \mathcal{S}}=\left(\alpha_{j}-\alpha_{i}\right) w_{i}$.

Step 5. If $\left\|z_{i j}^{h, \delta}\right\|>4 C\left(\delta+\varepsilon_{h}\right) / \sqrt{\alpha_{j}}$, then take $k=i-1$.

Step 6. Otherwise, repeat with $i+1$ in place of $i$.

Step 7. Choose $n_{k}=\min \left\{n: e^{-\gamma_{1} 3^{n}} \leq\left(\delta+\varepsilon_{h}\right) / \sqrt{\alpha_{k}}\right\}$.

Step 8. Solve $x_{n_{k}, \alpha_{k}}^{h, \delta}$ using the iteration (3.6).

In the next section we consider an example to illustrate the above algorithm. The computational results provided endorse the reliability and effectiveness of our method. 


\section{Example}

In this section we consider an example satisfying the assumptions made in this paper and give the numerical illustration. Consider the operator $K F: L^{2}(0,1) \rightarrow L^{2}(0,1)$ where $K$ : $L^{2}(0,1) \rightarrow L^{2}(0,1)$ is defined by

$$
K(x)(t)=\int_{0}^{1} k(t, s) x(s) d s
$$

and $F: D(F) \subseteq L^{2}(0,1) \rightarrow L^{2}(0,1)$ defined by

$$
F(u):=\int_{0}^{1} k(t, s) u^{3}(s) d s
$$

where

$$
k(t, s)= \begin{cases}(1-t) s, & 0 \leq s \leq t \leq 1 \\ (1-s) t, & 0 \leq t \leq s \leq 1\end{cases}
$$

Then for all $x(t), y(t): x(t)>y(t)$ (see [7], Section 4.3):

$$
\langle F(x)-F(y), x-y\rangle=\int_{0}^{1}\left[\int_{0}^{1} k(t, s)\left(x^{3}-y^{3}\right)(s) d s\right](x-y)(t) d t \geq 0 .
$$

Thus the operator $F$ is monotone. The Fréchet derivative of $F$ is given by

$$
F^{\prime}(u) w=3 \int_{0}^{1} k(t, s)(u(s))^{2} w(s) d s .
$$

Let $V_{n}$ be a sequence of finite-dimensional subspaces of $X$ and let $P_{h}=P_{1 / n}$ denote the orthogonal projection on $X$ with range $R\left(P_{h}\right)=V_{n}$. We assume that $\operatorname{dim} V_{n}=n+1$ and $\left\|P_{h} x-x\right\| \rightarrow 0$ as $h \rightarrow 0$ for all $x \in X$. We choose the linear splines $\left\{v_{1}, v_{2}, \ldots, v_{n+1}\right\}$ in a uniform grid of $n+1$ points in $[0,1]$ as a basis of $V_{n}$.

Since $w_{i} \in V_{n}, w_{i}$ is of the form $\sum_{i=1}^{n+1} \lambda_{i} v_{i}$ for some scalars $\lambda_{1}, \lambda_{2}, \ldots, \lambda_{n+1}$. It can be seen that $w_{i}$ is a solution of (4.2) if and only if $\bar{\lambda}=\left(\lambda_{1}, \lambda_{2}, \ldots, \lambda_{n+1}\right)^{T}$ is the unique solution of

$$
\left(M_{n}+\alpha_{i} B_{n}\right) \bar{\lambda}=\bar{a},
$$

where

$$
\begin{gathered}
M_{n}=\left(\left\langle K v_{i}, K v_{j}\right\rangle\right), \quad i, j=1,2, \ldots, n+1, \\
B_{n}=\left(\left\langle v_{i}, v_{j}\right\rangle\right), \quad i, j=1,2, \ldots, n+1, \\
\bar{a}=\left(\left\langle P_{h} K^{*}\left(f^{\mathcal{\delta}}-K F\left(x_{0}\right)\right), v_{i}\right\rangle\right)^{T}, \quad i=1,2, \ldots, n+1 .
\end{gathered}
$$


Observe that $z_{i j}^{h, \delta}$ in Step 4 of algorithm is again in $V_{n}$ and hence $z_{i j}^{h, \delta}=\sum_{k=1}^{n+1} \mu_{k}^{i j} v_{k}$ for some $\mu_{k}^{i j}, k=1,2, \ldots, n+1$. One can see that for $j<i, z_{i j}^{h, \delta}$ is a solution of

$$
\left(P_{h} K^{*} K P_{h}+\alpha_{j} I\right) z_{i j}^{h, \delta}=\left(\alpha_{j}-\alpha_{i}\right) w_{i}
$$

if and only if $\overline{\mu^{i j}}=\left(\mu_{1}^{i j}, \mu_{2}^{i j}, \ldots, \mu_{n+1}^{i j}\right)^{T}$ is the unique solution of

$$
\left(M_{n}+\alpha_{j} B_{n}\right) \overline{\mu^{i j}}=\bar{b}
$$

where

$$
\bar{b}=\left(\left\langle\left(\alpha_{j}-\alpha_{i}\right) w_{i}, v_{i}\right\rangle\right)^{T} .
$$

Compute $z_{i j}^{h, \delta}$ till $\left\|z_{i j}^{h, \delta}\right\|>4 C\left(\delta+\varepsilon_{h}\right) / \sqrt{\alpha_{j}}$ and fix $k=i-1$. Now choose $n_{k}=\min \left\{n: e^{-\gamma_{1} 3^{n}} \leq\right.$ $\left.\left(\delta+\varepsilon_{h}\right) / \sqrt{\left(\alpha_{k}\right)}\right\}$.

Let $\xi^{n}=\left(\xi_{1}^{n}, \xi_{2}^{n}, \ldots, \xi_{n+1}^{n}\right), \eta^{n}=\left(\eta_{1}^{n}, \eta_{2}^{n}, \ldots, \eta_{n+1}^{n}\right), y_{n, \alpha_{k}}^{h, \delta}=\sum_{i=1}^{n+1} \xi_{i}^{n} v_{i}$ and $x_{n, \alpha_{k}}^{h, \delta}=$ $\sum_{i=1}^{n+1} \eta_{i}^{n} v_{i}$. Then from (3.5) we have

$$
\begin{aligned}
\left(P_{h} F^{\prime}\left(x_{n, \alpha_{k}}^{h, \delta}\right)+\frac{\alpha_{k}}{c}\right) \sum_{i=1}^{n+1}\left(\xi_{i}^{n}-\eta_{i}^{n}\right) v_{i}= & \sum_{i=1}^{n+1} \lambda_{i} v_{i}-\sum_{i=1}^{n+1} P_{h} F\left(x_{n, \alpha_{k}}^{h, \delta}\right) v_{i} \\
& +\frac{\alpha_{k}}{c} \sum_{i=1}^{n+1}\left(x_{0}\left(t_{i}\right)-\eta_{i}^{n}\right) v_{i}
\end{aligned}
$$

where $t_{1}, t_{2}, \ldots, t_{n+1}$ are the grid points.

Observe that $\left(y_{n, \alpha_{k}}^{h, \delta}-x_{n, \alpha_{k}}^{h, \delta}\right)$ is a solution of (3.5) if and only if $\left(\overline{\xi^{n}-\eta^{n}}\right)=\left(\xi_{1}^{n}-\eta_{1}^{n}, \xi_{2}^{n}-\right.$ $\left.\eta_{2}^{n}, \ldots, \xi_{n+1}^{n}-\eta_{n+1}^{n}\right)^{T}$ is the unique solution of

$$
\left(Q_{n}+\frac{\alpha_{k}}{c} B_{n}\right)\left(\overline{\xi^{n}-\eta^{n}}\right)=B_{n}\left[\bar{\lambda}-F_{h 1}+\frac{\alpha_{k}}{c}\left(X_{0}-\overline{\eta^{n}}\right)\right]
$$

where $Q_{n}=\left\langle F^{\prime}\left(x_{n, \alpha_{k}}^{h, \delta}\right) v_{i}, v_{j}\right\rangle, i, j=1,2, \ldots, n+1$

$$
F_{h 1}=\left[F\left(x_{n, \alpha_{k}}^{h, \delta}\right)\left(t_{1}\right), F\left(x_{n, \alpha_{k}}^{h, \delta}\right)\left(t_{2}\right), \ldots, F\left(x_{n, \alpha_{k}}^{h, \delta}\right)\left(t_{n+1}\right)\right]^{T}
$$

and $X_{0}=\left[x_{0}\left(t_{1}\right), x_{0}\left(t_{2}\right), \ldots, x_{0}\left(t_{n+1}\right)\right]^{T}$.

Further from (3.6) it follows that

$$
\left(P_{h} F^{\prime}\left(x_{n, \alpha_{k}}^{h, \delta}\right)+\frac{\alpha_{k}}{c}\right)\left(x_{n+1, \alpha_{k}}^{h, \delta}-y_{n, \alpha_{k}}^{h, \delta}\right)=P_{h}\left[z_{\alpha_{k}}^{h, \delta}-F\left(y_{n, \alpha_{k}}^{h, \delta}\right)+\frac{\alpha_{k}}{c}\left(x_{0, \alpha_{k}}^{h, \delta}-y_{n, \alpha_{k}}^{h, \delta}\right)\right] .
$$




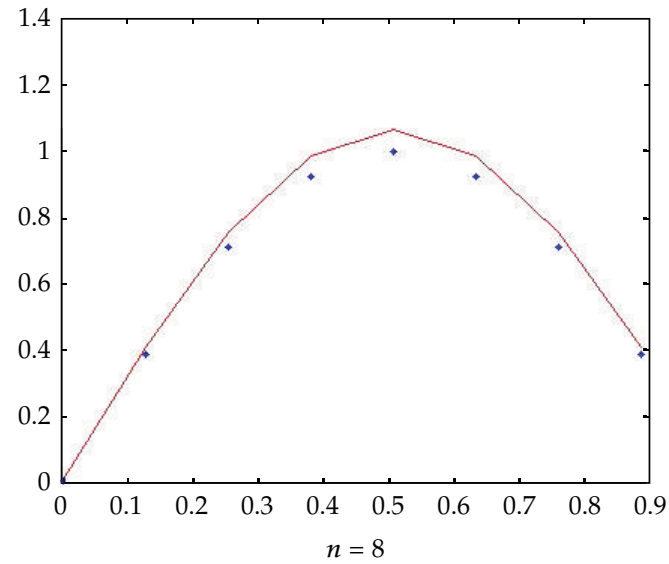

(a)

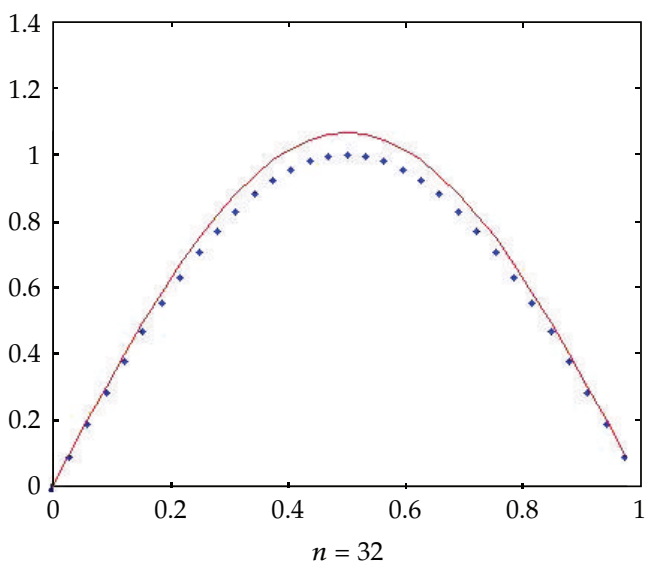

- Exact soln

— Approx. soln

(c)

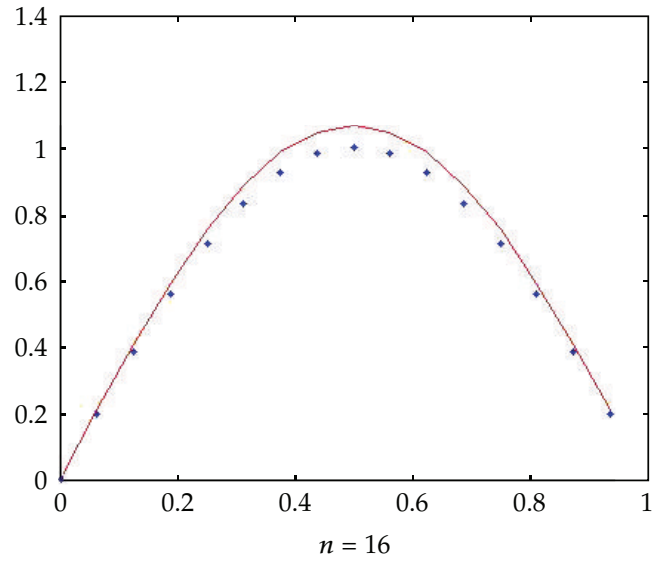

(b)

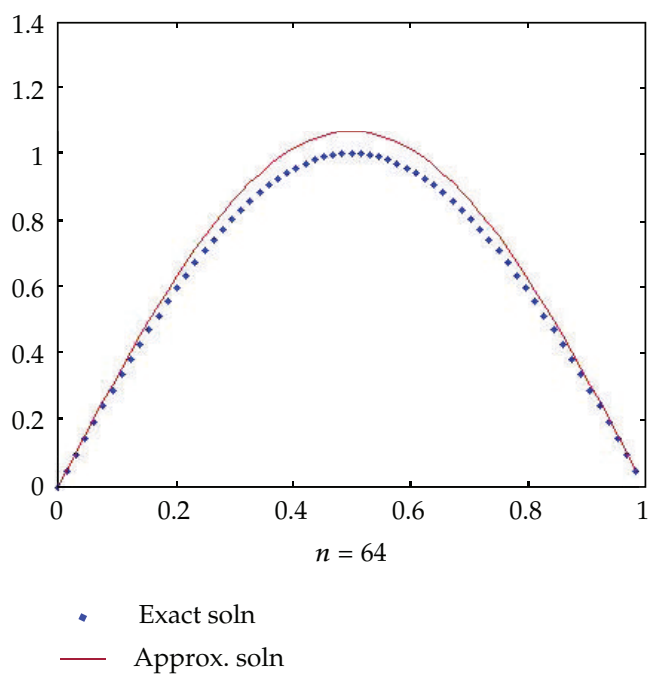

(d)

Figure 1: Curve of the exact and approximate solutions.

Thus $\left(x_{n+1, \alpha_{k}}^{h, \delta}-y_{n, \alpha_{k}}^{h, \delta}\right)$ is a solution of (5.14) if and only if $\left(\overline{\eta^{n+1}-\xi^{n}}\right)=\left(\eta_{1}^{n+1}-\xi_{1}^{n}, \eta_{2}^{n+1}-\right.$ $\left.\xi_{2}^{n}, \ldots, \eta_{n+1}^{n+1}-\xi_{n+1}^{n}\right)^{T}$ is the unique solution of

$$
\left(Q_{n}+\frac{\alpha_{k}}{c} B_{n}\right)\left(\overline{\eta^{n+1}-\xi^{n}}\right)=B_{n}\left[\bar{\lambda}-F_{h 2}+\frac{\alpha_{k}}{c}\left(X_{0}-\overline{\xi^{n}}\right)\right]
$$

where $F_{h 2}=\left[F\left(y_{n, \alpha_{k}}^{h, \delta}\right)\left(t_{1}\right), F\left(y_{n, \alpha_{k}}^{h, \delta}\right)\left(t_{2}\right), \ldots, F\left(y_{n, \alpha_{k}}^{h, \delta}\right)\left(t_{n+1}\right)\right]^{T}$.

\subsection{Numerical Example}

Example 5.1. To illustrate the method discussed in the above section, we consider the space $X=Y=L^{2}[0,1]$ and the Fredholm integral operator $K: L^{2}[0,1] \rightarrow L^{2}[0,1]$. The algorithm 


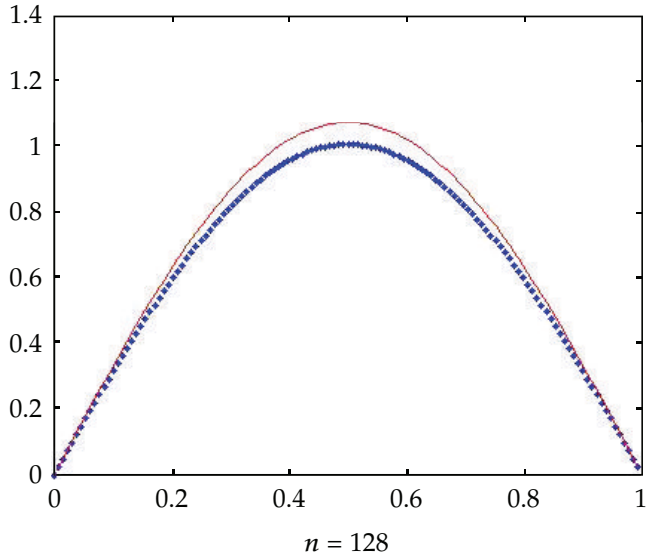

(a)

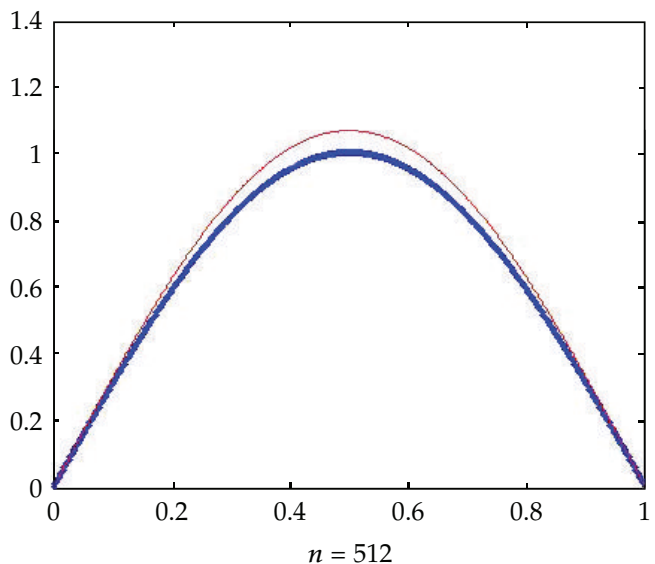

- Exact soln

- Approx. soln

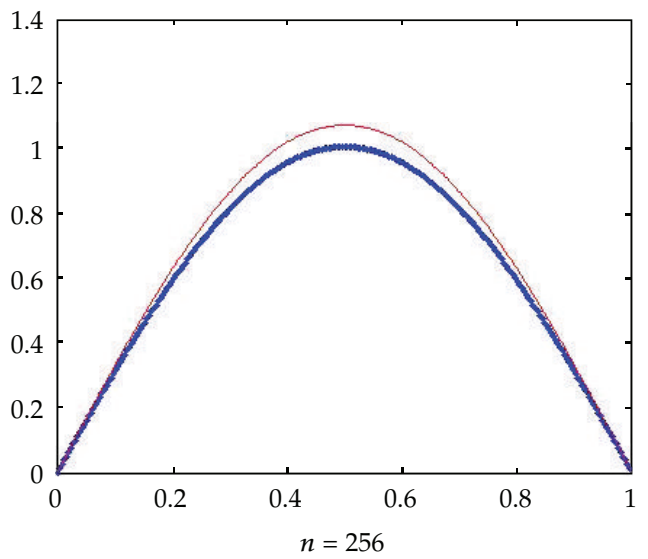

(b)

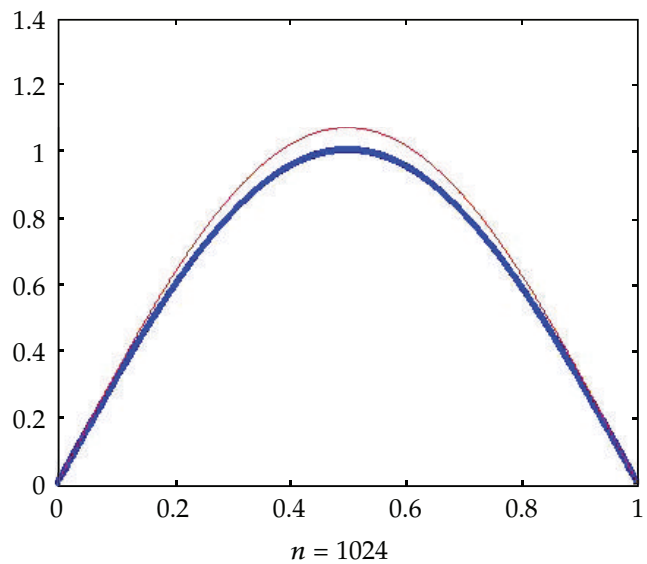

- Exact soln

— Approx. soln

(c)

(d)

Figure 2: Curve of exact and approximate solutions.

in Section 5 is applied by choosing $V_{n}$ as the space of linear splines in a uniform grid of $n+1$ points in $[0,1]$.

In our computation, we take $f(t)=\left(1 / 36 \pi^{2}\right)(27 \sin \pi t-\sin 3 \pi t)+(1 / 36 \pi)\left(27 t^{2} \cos \pi t-\right.$ $\left.3 t^{2} \cos 3 \pi t+6 t \cos 3 \pi t-3 \cos 3 \pi t-27 t \cos \pi t\right)$ and $f^{\delta}=f+\delta$. Then the exact solution

$$
\widehat{x}(t)=\sin \pi t .
$$

We use

$$
x_{0}(t)=\sin \pi t+\frac{3}{4 \pi^{2}}\left(1+t \pi^{2}-t^{2} \pi^{2}-\cos ^{2}(\pi t)\right)
$$


Table 1

\begin{tabular}{llccc}
\hline$n$ & $k$ & $\alpha_{k}$ & $\left\|x_{k}-\widehat{x}\right\|$ & $\left\|x_{k}-\widehat{x}\right\| /\left(\delta+\varepsilon_{h}\right)^{1 / 2}$ \\
\hline 8 & 4 & 0.1790 & 0.0363 & 0.1388 \\
16 & 4 & 0.1729 & 0.0432 & 0.1669 \\
32 & 4 & 0.1714 & 0.0450 & 0.1742 \\
64 & 4 & 0.1710 & 0.0455 & 0.1761 \\
128 & 4 & 0.1709 & 0.0456 & 0.1765 \\
256 & 4 & 0.1709 & 0.0456 & 0.1767 \\
512 & 4 & 0.1709 & 0.0456 & 0.1767 \\
1024 & 4 & 0.1709 & 0.0456 & 0.1767 \\
\hline
\end{tabular}

as our initial guess, so that the function $x_{0}-\hat{x}$ satisfies the source condition

$$
x_{0}-\widehat{x}=F^{\prime}(\widehat{x}) 1=\varphi_{1}\left(F^{\prime}\left(x_{0}\right)\right) G\left(x_{0}, \widehat{x}\right),
$$

where $\varphi_{1}(\lambda)=\lambda$. Thus we expect to have an accuracy of order at least $O\left(\left(\delta+\varepsilon_{h}\right)^{1 / 2}\right)$.

We choose $\alpha_{0}=(1.5) \delta^{2}, \mu=1.5, \delta=0.0667=c, \varepsilon_{h}=1 / 10 n^{2}, \rho=0.19, \gamma_{\rho}=0.8173$, and $g\left(\gamma_{\rho}\right)=0.54$ approximately. For all $n$ the number of iteration $n_{k}=3$ in this example. The results of the computation are presented in Table 1. The plots of the exact and the approximate solution obtained are given in Figures 1 and 2.

\section{Acknowledgment}

M. E. Shobha thanks National Institute of Technology Karnataka India, for the financial support.

\section{References}

[1] H. W. Engl, M. Hanke, and A. Neubauer, Regularization of Inverse Problems, Kluwer Academic, Dodrecht, The Netherlands, 1993.

[2] S. George and A. I. Elmahdy, "A quadratic convergence yielding iterative method for nonlinear illposed operator equations," Computational Methods in Applied Mathematics, vol. 12, p. 32, 2012.

[3] B. Kaltenbacher, A. Neubauer, and O. Scherzer, Iterative Regularisation Methods for Nolinear Ill-Posed Porblems, Walter de Gruyter, Berlin, Germany, 2008.

[4] S. George, “Newton-Tikhonov regularization of ill-posed Hammerstein operator equation," Journal of Inverse and Ill-Posed Problems, vol. 14, no. 2, pp. 135-145, 2006.

[5] S. George and M. T. Nair, "A modified Newton-Lavrentiev regularization for nonlinear ill-posed Hammerstein-type operator equations," Journal of Complexity, vol. 24, no. 2, pp. 228-240, 2008.

[6] P. Mahale and M. T. Nair, "A simplified generalized Gauss-Newton method for nonlinear ill-posed problems," Mathematics of Computation, vol. 78, no. 265, pp. 171-184, 2009.

[7] E. V. Semenova, "Lavrentiev regularization and balancing principle for solving ill-posed problems with monotone operators," Computational Methods in Applied Mathematics, vol. 10, no. 4, pp. 444-454, 2010.

[8] S. George and M. Kunhanandan, "An iterative regularization method for ill-posed Hammerstein type operator equation," Journal of Inverse and Ill-Posed Problems, vol. 17, no. 9, pp. 831-844, 2009.

[9] S. Pereverzev and E. Schock, "On the adaptive selection of the parameter in regularization of ill-posed problems," SIAM Journal on Numerical Analysis, vol. 43, no. 5, pp. 2060-2076, 2005.

[10] I. K. Argyros and S. Hilout, "A convergence analysis for directional two-step Newton methods," Numerical Algorithms, vol. 55, no. 4, pp. 503-528, 2010. 
[11] S. George and M. E. Shobha, Two Step Newton Tikhonov Methods for Hammerstein Equations (communicated).

[12] M. T. Nair and P. Ravishankar, "Regularized versions of continuous Newton's method and continuous modified Newton's method under general source conditions," Numerical Functional Analysis and Optimization. An International Journal, vol. 29, no. 9-10, pp. 1140-1165, 2008.

[13] A. G. Ramm, A. B. Smirnova, and A. Favini, "Continuous modified Newton's-type method for nonlinear operator equations," Annali di Matematica Pura ed Applicata. Series IV, vol. 182, no. 1, pp. 37-52, 2003. 


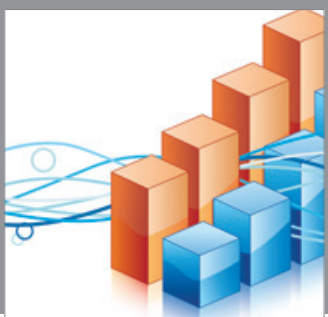

Advances in

Operations Research

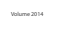

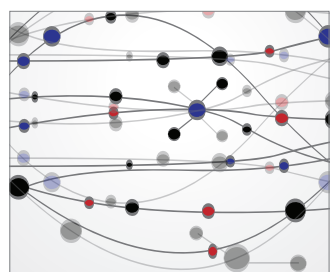

\section{The Scientific} World Journal
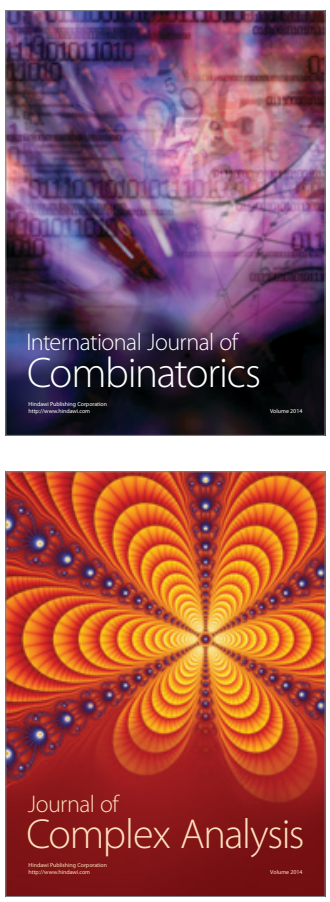

International Journal of

Mathematics and

Mathematical

Sciences
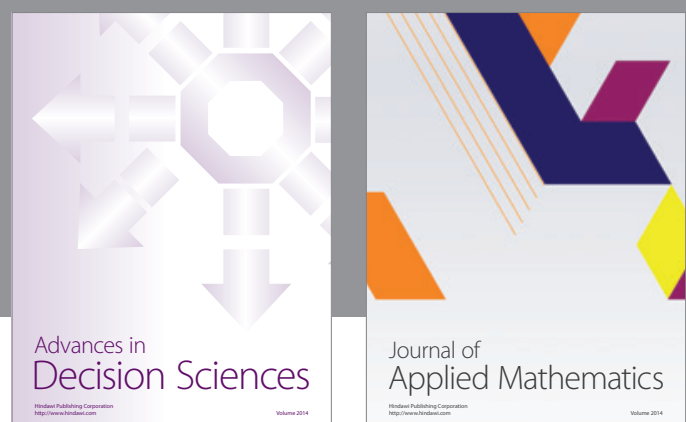

Journal of

Applied Mathematics
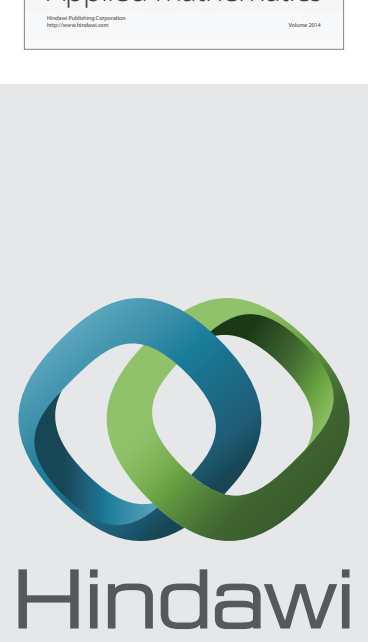

Submit your manuscripts at http://www.hindawi.com
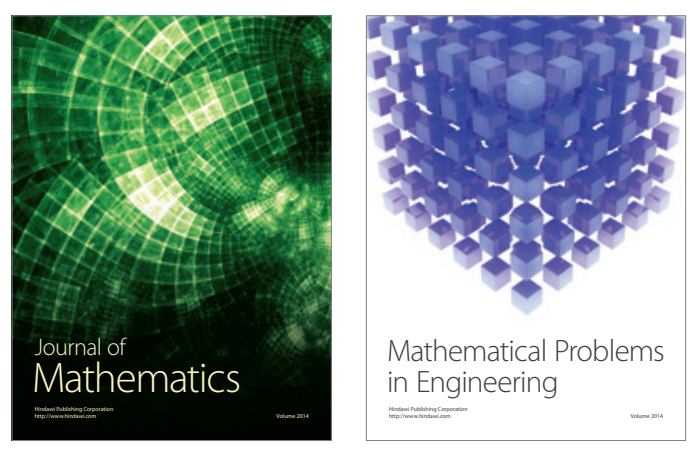

Mathematical Problems in Engineering
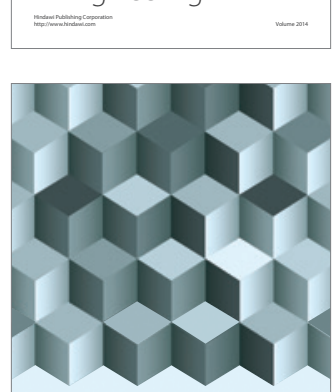

Journal of

Function Spaces
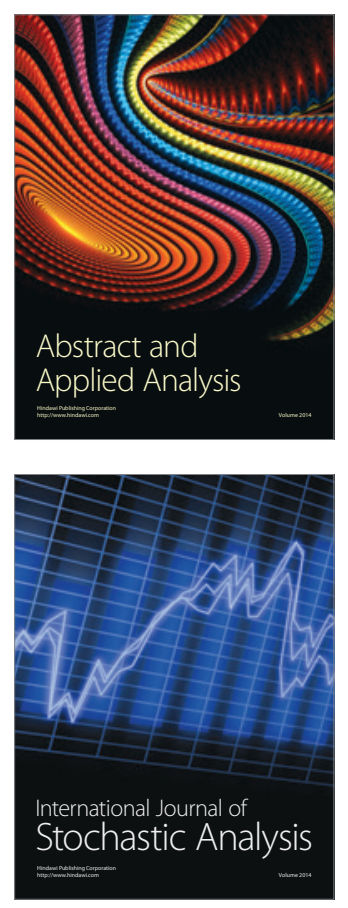

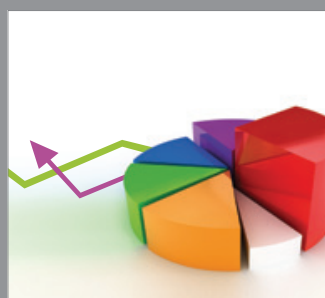

ournal of

Probability and Statistics

Promensencen
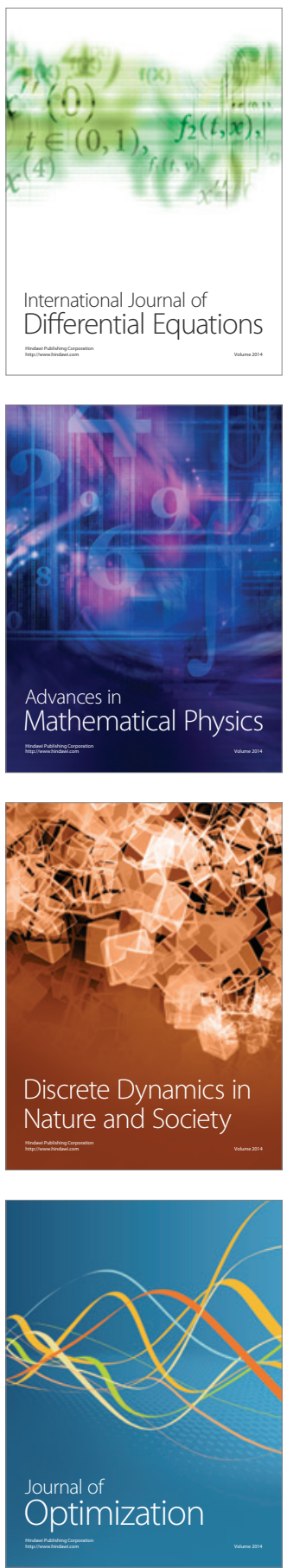